\title{
A New Method of Ambiguity Resolution for Triple-Frequency GPS PPP
}

\author{
Xiao-Feng LU ${ }^{1, a}$, Sheng-Li ZHANG ${ }^{1}$ and Ya-Chuan BAO ${ }^{2}$ \\ ${ }^{1}$ State Key Laboratory of Integrated Service Networks, Xidian University, Xi'an China \\ ${ }^{2}$ State Key Laboratory of Satellite Navigation System and Equiment Technology, Shijiazhuang, Hebei, 050081, China
}

\begin{abstract}
At present, reliable ambiguity resolution in GPS precise point positioning (PPP) can be achieved through the traditional model called "EWL-WL-NL". In this paper, we proposed a new model of ambiguity resolution,"WL-WL-WL", where making use of linear independence of coefficient vector of wide-lane combination. Firstly, using the MelbourneWubbena combination observable on L2 and L5, we could resolve extra-wide-lane ambiguity instantaneously. Then, the resolved unambiguous extra-wide-lane carrier-phase assists wide-lane ambiguity resolution (AR). Three wide-lane combinations whose coefficient vectors are linearly independent are chosen to compose one full-rank matrix so that the three narrow-lane ambiguity resolution can be achieved. As a result, with the triple-frequency signals, the correctness rate of narrow-lane ambiguity resolution achieves $90 \%$ within 60 s, in contrast to only $63 \%$ within 180 s in dual-frequency PPP. Therefore, we demonstrate that triple-frequency PPP has the potential to achieve ambiguity-fixed solutions within a few minutes and the efficiency of ambiguity resolution in triple-frequency PPP is higher.
\end{abstract}

\section{Introduction}

To achieve an accuracy comparable to that of relative positioning, it is necessary to resolve integer-cycle carrierphase ambiguities. This is straightforward in GPS relative positioning, which eliminates uncelebrated non-integer satellite and receiver carrier-phase hardware biases/delays (UPDs) through double differencing [1]. However, these biases will make it difficult to get integer ambiguities which make the integer ambiguity parameter loose integer property $[2,3]$. One approach computing the FCBs for GPS PPP ambiguity resolution is to use undifferenced ambiguities estimate FCBs over a network of reference stations, and then the FCB estimates are provided for PPP clients to carry out ambiguity resolution at a single station [4-7].

Traditional Three-carrier ambiguity resolution (TCAR) and dual-carrier ambiguity resolution for PPP have been demonstrated that its positional accuracy will be improved substantially if its ambiguity is fixed [8-10]. However, at present, with dual-frequency global positioning system (GPS) measurements, the sufficiently precise narrow-lane ambiguity estimates can hardly be obtained using less than 15 min of measurements [11] because wide-lane ambiguity resolution requires around $20 \min [8]$. With the advent of triple-frequency signals, rapid re-convergences within $5 \mathrm{~s}$ to ambiguity-fixed solutions in real-time PPP are achieved at an over $95 \%$ success rate if we can precisely propagate ionospheric delay estimates [12].

During the last few years, it has been demonstrated that multi-frequency satellite signals from Global Navigation Satellite System (GNSS) will assist rapid TCAR/CIR (cascade integer resolution) in relative positioning [13]. While for multi-frequency PPP, there are only a limited number of studies. For example, Jianghui Geng formulates a series of ionosphere-free observables combination which are composed of both pseudorange and carrier-phase. With these combinations, it propose a new AR model, "extrawide-lane, wide-lane, narrow-lane (EWL-WL-NL)", which achieve the decimeter-level positioning accuracy.

According to the studies above, we propose a new model of ambiguity resolution,"WL-WL-WL", where making use of linear independence of coefficient vector of wide-lane combination. This paper is organized as follows: firstly, introducing the method estimating FCBs; Secondly, formulating the combination observables; finally, showing the performance of triple-frequency PPP with ambiguity resolution.

\section{Methodology}

In this section, we briefly introduce the methods for GPS PPP to estimate FCBs, and then introduce the traditional model of ambiguity resolution and the new model, "WLWL-WL", of ambiguity resolution proposed in this paper. 


\subsection{Estimation of Fractional-Cycle Biases}

In this paper, we use the following equation to denote raw GPS measurements on frequency $i(I=1,2,5)$ from station $r$ to satellite s. In this equation, some un-modeled errors are ignored such as multipath effects and some low influential factors are also ignored such as high-order ionospheric delay.

$$
P_{i r}^{s}=\rho_{r}^{s}+\mu_{i} I_{1}+T+b_{i r}-b_{i}^{s}
$$

$$
L_{i r}^{s}=\rho_{r}^{s}-\mu_{i} I_{1}+T-\lambda_{i}\left(N_{i r}^{s}+B_{i r}-B_{i}^{s}\right)
$$

where the subscripts $r$ denotes the receiver; $I=1,2,5$ denotes the L1, L2 and L5 frequencies, respectively. $P_{i r}^{s}$ and $L_{i r}^{s}$ are the un-differenced code and phase observation vector; $\rho_{r}^{s}$ is the vector of geometric distances between satellites and a receiver; ${ }^{I_{1}}$ is the first-order ionospheric delay on frequency $L_{1}$ with $\mu_{i}=f_{1}^{2} / f_{i}^{2} ; \lambda_{i}$ is the carrier phase wavelength; $f$ is frequency; $\mathrm{T}$ is the Tropospheric Delays; $N_{i r}^{s}$ is the integer ambiguity vector(cycle); $b_{i r}$ is the receiver code biases and $b_{i}^{s}$ is satellite code bias vector; $B_{i r}$ is the receiver FCB and $B_{i}^{s}$ is the satellite FCB vector;

In PPP, un-differenced float ambiguities can be formulated as the following equation.

$$
B_{r}^{s}=N_{r}^{s}+\left\langle B_{r}\right\rangle-\left\langle B^{s}\right\rangle
$$

$$
\text { where } B_{r}^{s} \text { is float un-differenced ambiguities; }\langle\cdot\rangle
$$

denotes the operation of estimating the fractional part; $N_{r}^{s}$ is the integer ambiguity which contains the integer parts of receiver- and satellite-specific hardware biases.

According to Eq.(6) and Eq.(7) in [10], we can obtain the approximate extra-wide-lane FCBs and wide-lane FCBs for all receivers and satellites using a network of reference stations and then deliver the FCBs to PPP clients. Once PPP clients receive the extra-wide-lane and wide-lane FCBs, ambiguity resolution for triple-frequency PPP can be attempted.

\subsection{Triple-Frequency PPP with Ambiguity Resolution}

In the paper, we propose a new ambiguity resolution model called "WL-WL-WL", and the ambiguity resolution is divided into three steps.

Firstly, we can obtain extra-wide-lane ambiguity through traditional method of ambiguity resolution.

$$
\begin{gathered}
L_{e w, \mathrm{r}}^{s}=\frac{f_{2} L_{2 r}^{s}-f_{5} L_{5 r}^{s}}{f_{2}-f_{5}}-\frac{f_{2} P_{2 r}^{s}+f_{5} P_{5 r}^{s}}{f_{2}+f_{5}} \\
=\lambda_{e w}\left(N_{e w, r}^{s}+\left\langle B_{e w, r}\right\rangle-\left\langle B_{e w}^{s}\right\rangle\right)
\end{gathered}
$$

where $L_{e w, \mathrm{r}}^{s}$ is combination observable; $\lambda_{e w}$ is extrawide-lane wavelength; $N_{e w, r}^{s}$ is extra-wide-lane integer ambiguity; $B_{e w, r}$ and $B_{e w}^{s}$ are the hardware biases within $L_{e w, \mathrm{r}}^{s}$.

Secondly, with the assist of ambiguity-fixed extra-widelane carrier-phase observables, we can obtain wide-lane ambiguity. For wide-lane combination, three linearly independent vector are chosen as coefficient vector and they are $(1,-1,0),(1,0,-1)$ and $(-2,-4,7)$, respectively. When the coefficient vector is $(1,-1,0)$, the wide-lane ambiguity can be obtained through traditional method.

$$
\left\{\begin{aligned}
L_{m r}^{s} & =\frac{f_{1}}{f_{1}-f_{2}} L_{1 r}^{s}-\frac{f_{2}}{f_{1}-f_{2}} L_{2 r}^{s} \\
L_{n r}^{s} & =\frac{f_{2}}{f_{2}-f_{5}} L_{2 r}^{s}-\frac{f_{5}}{f_{2}-f_{5}} L_{5 r}^{s}-\lambda_{e w} N_{e w, r}^{s} \\
P_{3 i}^{j} & =\frac{f_{1}^{2}}{f_{1}^{2}-f_{2}^{2}} P_{1 r}^{s}-\frac{f_{2}^{2}}{f_{1}^{2}-f_{2}^{2}} P_{2 r}^{s} \\
L_{w r}^{s} & =\frac{f_{1}}{f_{1}-f_{5}} L_{m r}^{s}-\frac{f_{5}}{f_{1}-f_{5}} L_{n r}^{s} \\
& =\rho_{r}^{s}+\lambda_{w} \frac{f_{1}}{f_{1}-f_{5}}\left(N_{w r}^{s}+\left\langle B_{w r}\right\rangle-\left\langle B_{w}^{s}\right\rangle\right)
\end{aligned}\right.
$$

where $L_{w r}^{s}$ is combination observable; ${ }^{\lambda} w$ is wide-lane wavelength; $N_{w r}^{s}$ is extra-wide-lane integer ambiguity; $B_{w r}$ and $B_{w}^{s}$ are the hardware biases within $L_{w r}^{s}$.

When the coefficient vector is $(1,0,-1)$, the wide-lane ambiguity resolution can be carried out with the following equation, that is

$$
P_{w r}^{s}=\frac{f_{1}^{2}}{f_{1}^{2}-f_{2}^{2}} P_{1 r}^{s}-\frac{f_{2}^{2}}{f_{1}^{2}-f_{2}^{2}} P_{2 r}^{s}
$$




$$
\left\{\begin{aligned}
L_{w a, r}^{s} & =\frac{f_{1}}{f_{1}-f_{5}} L_{1 r}^{s}-\frac{f_{5}}{f_{1}-f_{5}} L_{5 r}^{s} \\
L_{w b, r}^{s} & =\frac{f_{2}}{f_{2}-f_{5}} L_{2 r}^{s}-\frac{f_{5}}{f_{2}-f_{5}} L_{5 r}^{s} \\
L_{w 1, r}^{s} & =\frac{f_{1}}{f_{1}-f_{2}} L_{w a, r}^{s}-\frac{f_{2}}{f_{1}-f_{2}}\left(L_{w b, r}^{s}-\lambda_{e w} N_{\mathrm{ew}, r}^{s}\right) \\
& =\rho_{r}^{s}+\lambda_{w 1} \frac{f_{1}}{f_{1}-f_{2}}\left(N_{w 1, r}^{s}+\left\langle B_{w 1, r}\right\rangle-\left\langle B_{w 1}^{s}\right\rangle\right)
\end{aligned}\right.
$$

where

$$
\begin{aligned}
& \lambda_{w 1}=\frac{c}{f_{1}-f_{5}} \\
& \lambda_{e w}=\frac{c}{f_{2}-f_{5}} \\
& N_{w 1, r}^{s}=N_{1 r}^{s}-N_{5 r}^{s}+\left[B_{w 1, r}\right]+\left[B_{w 1}^{s}\right] \\
& N_{\mathrm{ew}, r}^{s}=N_{2 r}^{s}-N_{5 r}^{s}+\left[B_{\mathrm{ew}, r}\right]+\left[B_{e w}^{s}\right]
\end{aligned}
$$

and $P_{w r}^{s}$ is ionosphere-free pseudorange combination; $L_{w 1, r}^{s}$ is wide-lane carrier phase measurements; $N_{w 1, r}^{s}$ is the integer wide-lane ambiguity; $N_{\mathrm{ew}, r}^{s}$ is the integer extrawide-lane ambiguity; $B_{w 1, r}$ and $B_{w 1}^{s}$ are the hardware biases within $L_{w 1, r}^{s} ; B_{\mathrm{ew}, r}$ and $B_{e w}^{s}$ are the hardware biases within extra-wide-lane combination observable; From the equation, we can easily find that the wide-lane wavelength is about $0.75 \mathrm{~m}$ which is about 3.9 times to the wavelength on L1, which will facilitate rapid ambiguity resolution. Although its noise is amplified, the noise level is far less than its length, thereby showing that $N_{w 1, r}^{s}$ can be rapidly, if not instantaneously, fixed to an integer.

When the coefficient vector is $(-2,-4,7)$, the wide-lane ambiguity resolution can be carried out with the following equation, that is

$$
\left\{\begin{aligned}
L_{3 r}^{s}= & \frac{f_{5}^{2}}{f_{1}^{2}-f_{5}^{2}} L_{5 r}^{s}-\frac{f_{1}^{2}}{f_{1}^{2}-f_{5}^{2}} L_{1 r}^{s} \\
L_{w 2, r}^{s} & =L_{3 r}^{s}-\frac{\lambda_{1} f_{1}^{2}}{f_{1}^{2}-f_{5}^{2}} N_{w 1, r}^{s}-2 \lambda_{w 1} N_{w 1, r}^{s}-4 \lambda_{w 1} N_{e w, r}^{s} \\
& =\rho_{r}^{s}-\lambda_{w 1}\left(N_{w 2 r}^{s}+\left\langle B_{w 2, r}\right\rangle-\left\langle B_{w 2}^{s}\right\rangle\right)
\end{aligned}\right.
$$

where $N_{w 2 r}^{s}$ is the integer wide-lane ambiguity; $B_{w 2, r}$ and $B_{w 2}^{s}$ are the hardware biases within $L_{w 2, r}^{s}$. In this equation, the wide-lane wavelength is about $1.72 \mathrm{~m}$, which is about 9 times to the wavelength on L1, which make it easily to fix ambiguity. The Eq.(6) is taken as pseudorange within the equation.

Thirdly, once wide-lane ambiguity resolution is completed, the fixed wide-lane ambiguity can be taken as known quantity to assist in narrow-lane ambiguity resolution. The equation of narrow-lane ambiguity resolution is as follows:

$$
\left(\begin{array}{l}
N_{1} \\
N_{2} \\
N_{3}
\end{array}\right)=\left[\begin{array}{ccc}
1 & -1 & 0 \\
1 & 0 & -1 \\
-2 & -4 & 7
\end{array}\right]^{-1}\left(\begin{array}{l}
N_{(1,-1,0)} \\
N_{(1,0,-1)} \\
N_{(-2,-4,7)}
\end{array}\right)
$$

\section{Results and Discussion}

In this paper, the processing data resulting from IGS were used to estimate FCBs and fix ambiguity. Each epoch is 30 s. There are five stations selected totally and they are 'jfng', 'wuh2', 'gmsd', 'mizu' and 'unb3', respectively. The station ' $\mathrm{j}$ fng' was selected as the reference station, and 'unb3' was selected as the PPP client. This paper presents the correctness rate of triple-frequency PPP ambiguity resolution proposed in this paper and comparing the performance of the method proposed in this paper with performance of dual-frequency PPP ambiguity resolution proposed in [12].
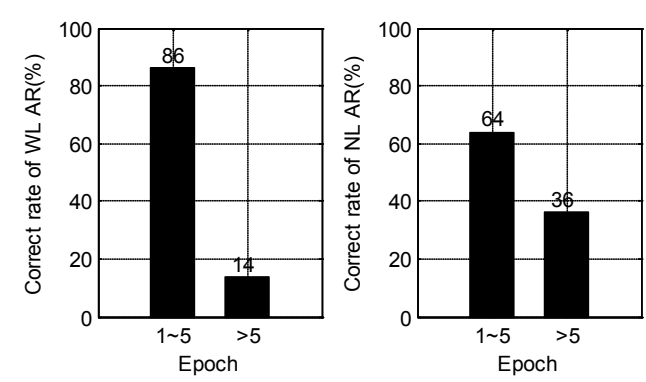

Fig. 1 Correct Rate of Wide-Lane(Wl) And Narrow-Lane(Nl) Ambiguity Resolution(Ar) In Dual-Freqnency Ppp.
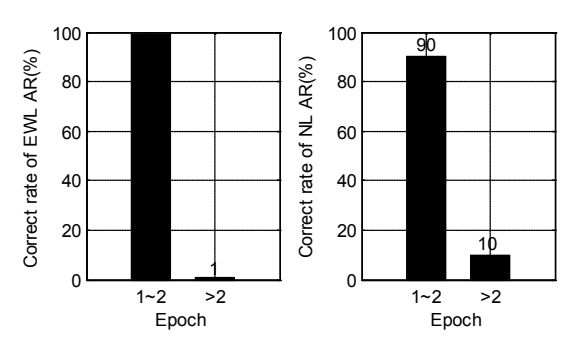


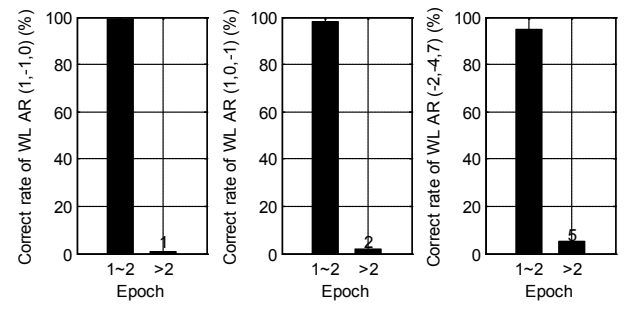

Fig.2 Correct Rate of Extra-Wide-Lane(Ewl), Three WideLane(Wl) And Narrow-Lane(Nl) Ambiguity Resolution (Ar) In Triple-Frequency Ppp And N1 Frequency Is L1.

Figure 1 shows the correctness rate of dual-frequency PPP ambiguity resolution proposed in [12]. Figure 2 shows the correctness rate of triple-frequency PPP ambiguity resolution proposed in this paper including the extra-widelane, wide-lane and narrow-lane ambiguity. And for narrow-lane-ambiguity, we show the result on L1. According to figure 1, the correctness rates of narrow-lane ambiguity resolution of dual-frequency PPP can only reach $64 \%$ over 150 -second observation periods. However, a $90 \%$ correctness rate can be achieved in triple-frequency PPP within 60s. It's obviously that the method proposed by this paper is superior to dual-frequency ambiguity resolution.

Compared with traditional ambiguity resolution, the method in this paper has two superiority. First, in the traditional method, it's necessary to estimate EWL, WL, NL FCBs. According to [2], we can find that NL FCBs are not stable and it has to be estimated every $15 \mathrm{~min}$ at least, which need heavy computational effort at reference network and increasing network burden. However, it's unnecessary to estimate NL FCBs. Therefore, we can conclude that the ambiguity resolution proposed by this paper outperform the traditional triple-frequency ambiguity resolution within certain limits.

\section{Conclusions}

In this paper, we proposed a new method for triplefrequency GPS PPP in order to achieve rapid ambiguity resolution with a single receiver in real time. The key section of this method is to fix three linearly independent wide-lane combinations ambiguity with the assist of the ambiguity-fixed extra-wide-lane carrier-phase on L2 and L5. According to analysis, the correctness rate of narrowlane ambiguity resolution in triple-frequency PPP can reach $90 \%$ within $60 \mathrm{~s}$, in contrast to 150 s for $64 \%$ correctness rate in dual-frequency PPP. According to analysis, we can obtain two effective conclusions:(1) With the data of triplefrequency signal, the convergence rate of ambiguity solution accelerating obviously; (2) With appropriate extrawide-lane and wide-lane combination, correctness rate of fixing narrow-lane ambiguity with triple-frequency signals is higher that with dual-frequency signals.

Finally, we can conclude that the ambiguity resolution efficiency in triple-frequency PPP is much higher than that in dual-frequency PPP. However, the wide-lane combinations in this paper may not be the optimal, which need to be researched deeply in future work.

\section{Acknowledgement}

This work was supported by National Natural Science Foundation of China (Grant No. 61371127, 61471361, 61401330, 61572389), EU FP7 Project CROWN (PIRSESGA-2013-610524), National High-tech R\&D Program of China (863 Program) (Grant No. 2015AA01A705), and 111 Project in Xidian University of China (Grant No.B08038).

\section{References}

1. Yanming Feng, Chris Rizos. GPS Solut 13 43-56 (2009)

2. Jianghui Geng, Chuang Shi, Maorong Ge, Alan H.Dodson, Yidong Lou, Qile Zhao, Jiangnan Liu. J Geod 86 579-589 (2012)

3. Jianghui Geng, Xiaolin Meng, Alan H. Dodson, Felix N. Teferle. J Geod 84 569-581 (2010)

4. Xingxing Li, Maorong Ge, Hongping Zhang,Jens Wichert. J Geod 87 405-416 (2013)

5. Shengfeng Gu, Yidong Lou, Chuang Shi, Jingnan Liu. J Geod 89 979-992 (2015)

6. Pan li, Xiaohong Zhang, Xiaodong Ren, Xiang Zuo, Yuming Pan. GPS Solut 2015

7. Jianghui Geng, Yehuda Rock. J Geod 90 379-396 (2016)

8. Jianghui Geng, F. N. Teferle, C. Shi, X. Meng, A. H. Dodson, J. Liu. GPS Solut 13 263-270 (2009)

9. M. Ge, G. Gendt, M. Rothacher, C. Shi, J. Liu. J Geod 82 389-399 (2008)

10. Yihe Li, Yang Gao, Junbo Shi. J Geod 90 437-450 (2016)

11. J. Geng, F.N. Teferle, X. Meng, A.H. Dodson. Adv. Space. Res 47 1664-1673 (2011)

12. Jianghui Geng, Yehuda Rock. J Geod 87 449-460 (2013)

13. Qile Zhao, Zhiqiang Dai, Zhigang $\mathrm{Hu}$, Binzi Sun, Chuang Shi, Jiangnan Liu. GPS Solut 19 589-599 (2015)

14. Simon Banville. J Geod 90 487-496 (2016) 